\title{
Rocking Curve Imaging for Diamond Radiator Crystal Selection
}

\author{
Guangliang Yang ${ }^{1}$, Richard Jones ${ }^{2}$, Franz Klein ${ }^{3}$, Ken Finkelstein ${ }^{4}$, Ken Livingston ${ }^{1}$ \\ ${ }^{1}$ Department of Physics and Astronomy, University of Glasgow, Glasgow, UK G12 8QQ. \\ ${ }^{2}$ University of Connecticut unit 3046, 2152 Hillside Rd., Storrs, CT, USA 06269-3046. \\ ${ }^{3}$ Catholic University of America, 620 Michigan Ave., N.E. Washington, DC 20064. \\ ${ }^{4}$ Cornell High Energy Synchrotron Source, Cornell University, Ithaca, NY, USA $14853 .$.
}

\begin{abstract}
The combination of low atomic number, high crystal packing density, and very high Debye temperature makes diamond the best material for use as a bremsstrahlung radiator in the coherent bremsstrahlung (CB) process, a process that is uniquely suited for generating highly polarized highenergy photon beams for photonuclear experiments. The crystal quality of the diamond radiator has a vital effect on the polarization and other properties of the photon beam and the best large-area diamond monocrystals currently available, both natural and synthetic, contain many defects that can degrade their performance as $\mathrm{CB}$ radiators. The diamonds used for this study were synthetic type $\mathrm{Ib}$ samples produced through the HPHT process by the firm Element Six. They were examined using the double crystal rocking curve imaging method in a synchrotron X-ray beam. Dislocation densities were calculated from the measured rocking curve peak position maps in the way proposed by Ferrari et al[1]. It is shown that dislocation is one major defect that affects the rocking curve width in local regions. The most significant contribution to the whole-crystal rocking curve width for thin crystals is the systematic variation of the peak position across its surface. This is interpreted in terms of a large-scale bending of the entire crystal. Data supporting this interpretation are presented, and possible explanations for the bending and methods for its mitigation are discussed.
\end{abstract}




\section{Introduction}

Diamond radiators are widely used to generate highly polarized photon beams through the coherent bremsstralung process $(\mathrm{CB})$ at many laboratories in the world, including Jefferson Lab, MAX_lab, and MAMI [2,3,4]. The combination of low atomic number, high crystal packing density, and very high Debye temperature makes diamond the best material for CB radiators [5]. The diamond crystal quality has a vital effect on the polarization of the photon beam; only those crystals that are of very high quality are suitable to be used as a photon radiator [5]. The most important measure of diamond quality that affects $\mathrm{CB}$ radiator performance is its mosaic spread, the deviation from parallel of the local normal to a set of crystal planes throughout the crystal volume. This requirement depends on the energy of the $\mathrm{CB}$ beam, but in general mosaic spreads greater than 10 times the natural width must be avoided. This requirement applies to the entire crystal, which must be on the order of $50 \mathrm{~mm}^{2}$ in area and less than 50 microns in thickness. The diamond purity requirements for the $\mathrm{CB}$ application are relatively modest (a few ppm of nitrogen is tolerable), but the combination of large area and good crystal quality described above rules out natural diamonds as an affordable source for CB radiators.

Today's synthetic diamond growth techniques can produce very high quality diamond for various applications; however diamonds with sufficiently large high quality and large area for use as $\mathrm{CB}$ radiators are still not readily available and require a prescreening procedure to be carried out, in cooperation with the manufacturer, prior to the final machining process. This is because the diamonds made by HPHT or CVD technologies are not completely free of defects, and the defect distribution is not uniform, either across the face of a single crystal or between different crystals 
produced by the same vendor. Therefore, it is our concern to understand the most important factors that contribute to a large mosaic spread in synthetic crystals, and to develop efficient non-destructive methods to select samples for post-processing that meet the requirements for use as diamond radiators.

High resolution $\mathrm{x}$ ray rocking curve and topograph measurements are the most efficient methods for assessing the mosaic spread of diamond samples. X-rays are scattered by atomic electrons, whereas in coherent bremsstrahlung the high-energy electrons scatter from the total charge distribution of the lattice, but both processes are governed by the same crystal form factor. The X-ray rocking curve width for an appropriate set of planes translates directly into a lower bound for the width of the primary coherent peak in the $\mathrm{CB}$ spectrum. In our previous diamond selection studies [6], a single detector was used to collect the data, which integrates the scattering from the sample over all angles within its aperture. Measurements with a single detector can only yield information about the overall quality of the region exposed to the X-ray beam. However a diamond crystal may appear to have an acceptable overall quality, but still have limited regions on its surface the crystal quality is very poor. If the electron beam in the $\mathrm{CB}$ source were to strike such a bad region, it would spoil the quality of the polarization of the generated photon beam. Therefore it is of interest to know how the crystal quality varies across the surface of the sample. In this paper, we give details about how we use rocking curve images to assess the diamond quality and to investigate the nature of the major contributors to the rocking curve width for a few sample diamonds. 


\section{Experimental setup}

The diamonds were synthetic type $\mathrm{Ib}(001)$ oriented plates and were purchased from Drukker (now renamed Element Six). The rocking curve imaging measurements were carried out at the Cornell High Energy Synchrotron Source (CHESS), Ithaca NY on the $\mathrm{C} 1$ beam line. The sample was rotated in a four-axis goniometer with arc-second resolution in the Bragg angle, oriented so that the scattering takes place in the vertical plane. The sample was positioned $14.5 \mathrm{~m}$ from the bending magnet source. Upstream of the hutch, the white beam from the bending magnet passed through a two-bounce silicon monochromator, which served both to select $15 \mathrm{KeV} \mathrm{X}$-rays from the white beam and to expand the beam by a factor 8 in the vertical direction. By using the $(3,3,1)$ planes of the silicon monochromator together with the $(2,2,0)$ planes of the diamond sample, a nearly perfect non-dispersive match was achieved. A single detector with fast readout was used during setup to align the crystal for rocking curve measurements, and then a pixel detector was used to scan through the rocking curve peak and image the crystal at each setting of the Bragg angle. The transverse resolution of the X-ray camera was determined using a template to be 50 microns r.m.s.

\section{Results}

The X-ray intensities for individual pixels were extracted from the image series for a given rocking curve scan, and the position and width of the peak for that pixel extracted using a Gaussian fit. Maps of the rocking curve width for the $(2,2,0)$ planes in two different diamond crystals are shown in Fig. 1. It can be seen that these diamond samples are locally nearly perfect over a substantial fraction of their area, in that their observed rocking curve widths are comparable to the natural width of 
diamond of $5 \mu \mathrm{rWHM}$ at $15 \mathrm{keV}$ for these planes. However the images also show some "hotspots" where the rocking curve width locally is much larger than its average for the entire crystal.

Apart from the rocking curve width map, the rocking curve peak position map also vields important information. In Fig. 2, maps of rocking curve width and peak position of are shown, both taken from the same scan of a diamond that is 20 microns in thickness for a scan of the $(2,2,0)$ planes. Scans of the same region taken with the $(2,-2,0)$ planes are qualitatively similar, and are not shown. It can be seen that for most of the region of this crystal, the rocking curve width is very small, indicating very good quality. However, the rocking curve peak position varies dramatically across the crystal, making it unsuitable for use as a radiator. This is because the electron beam size in a $\mathrm{CB}$ source is much larger than the dimensions of one pixel in the $\mathrm{CCD}$ camera. For example, the size of the electron beam used to produce the $\mathrm{CB}$ photon beam for the GlueX experiment at Jefferson Lab will be $0.8 \mathrm{~mm}$ r.m.s. by 0.3 mm r.m.s. [7]. The rocking curve of the region seen by the electron beam is the sum of all the rocking curves of pixels within the electron beam boundary weighted by the electron beam intensity profile. Thus the peak position variation makes the effective mosaic spread seen by the electron beam in this diamond much larger than the width observed for a single pixel in the CCD.

A smooth variation of the rocking curve peak position is explained most simply in terms of the mechanical bending of the crystal. Although bulk diamond is mechanically very rigid, a thin wafer can easily bend about an axis within parallel to its surface, either in response to stress from the mounting fixture or because of strain 
caused by defects. It has been pointed out by Albert[8] the lattice strain for high quality diamond could be very small, at the level of $10^{-6}$. From the very narrow rocking curve width in local regions, we conclude that the diamond depicted in Fig. 2 is of very high quality. Therefore, it is reasonable to suppose that the variation of the peak position is mainly caused by the crystal curvature, rather than variation in the value of the d-spacing across the crystal. This hypothesis can be tested by comparing two rocking curves taken with the same set of $(2,2,0)$ diamond planes, but rotated about the $(0,0,1)$ axis by 180 degrees.. Since this rearrangement reverses the shape of the rocking curve features that arise from curvature, while the features caused by variation in d-spacing should remain unchanged. The average and the difference of the two measurements yield the lattice parameter variation and lattice tilts, respectively. Our results confirmed that the effect of lattice parameter variations is negligible in our case, and that the variation of the rocking curve peak position is mainly caused by the crystal warping.

The crystal diffraction plane orientation can be determined by the measured rocking curve peak position, and the diffraction plane shape can be calculated from the diffraction plane orientation in the following way[1]. Consider the lattice displacement field $\mathbf{u}(\mathbf{r})$. We focus on its component along the direction of the surface normal $u_{z}(x, y)$ as a function of position $(x, y)$ in the plane of the sample surface. Local tilt angles $\theta_{x}, \theta_{y}$ of the $(0,0,1)$ planes of the physical crystal may be defined relative to to the $x, y$ plane in this coordinate system as follows.

$$
\begin{aligned}
& \tan \theta_{x}=\frac{\partial u_{z}}{\partial x} \\
& \tan \theta_{y}=\frac{\partial u_{z}}{\partial y}
\end{aligned}
$$


In our measurement, we interpret the offset of the $(2,2,0)$ rocking curve peak angle from its average position as the function $\theta_{x}(x, y)$, and of the $(2,-2,0)$ planes as $\theta_{y}(x, y)$. The two dimensional gradient of $u_{z}(x, y)$ in the surface plane is defined as follows.

$$
\mathbf{V}(x, y)=\mathbf{i} \frac{\partial u_{x}}{\partial x}+\mathbf{j} \frac{\partial u_{y}}{\partial y}
$$

Consider the integral of $\mathbf{V}(x, y)$ along a closed path around some point on the sample surface. In a perfect crystal, the integral is zero everywhere. In a crystal containing dislocations, the integral is equal to the total z-component of the Burgers vectors of dislocations within the closed path[9].

$$
\oint \mathbf{V} \cdot d \mathbf{l}=\sum_{i} b_{z}^{i}
$$

If the dislocation density is zero, we can calculate the lattice displacement $u_{z}$ from its gradient field by using the relation

$$
u_{z}(x, y)=\int_{0,0}^{x, y} \mathbf{V}(x, y) \cdot d \mathbf{l}
$$

where the origin in the $x, y$ plane represents the point in the crystal where the displacement field is defined to be zero. The dislocation density for the 20 micron diamond calculated using Eq. (4) shows that the dislocation density is well below $10^{4} / \mathrm{cm}^{2}$ for most of the region shown in Fig. 2. In local regions where the dislocation density is much higher, the measured rocking curve width is also much larger, indicating that dislocation is one of the main reasons for rocking curve broadening in those areas

In order to use Eq. (5) to calculate the crystal curvature, the dislocation density should be zero. However, in cases where the dislocation density is low and the crystal 
deformation is very large, we can neglect the effects of dislocation and calculate the crystal shape from the $(2,2,0)$ and $(2,-2,0)$ rocking curve data using Eq. (5) .

The calculated crystal shape for the 20 micron crystal is shown in Fig. 3a. As a cross check, it is possible to go the other way and use the calculated crystal shape to compute what it should produce for rocking curves. . This cross-check was carried out for the 20 micron crystal, and the simulated result agrees well with the measured results, as shown in Fig. 3b.

\section{Conclusions}

From the results obtained for the 20 micron diamond, it is clear that crystal warping is a major issue that needs to be understood and resolved before crystals of these dimensions can be used as CB radiators. The crystal warping may be caused by nonuniformly distributed defects, by surface damage that occurs during the lapping and polishing steps in the diamond wafer production, or perhaps by the stress induced by crystal mounting fixture. Using a thicker crystal will help to mitigate some of these effects, but unfortunately, the optimum $\mathrm{CB}$ performance requires the diamond radiator to be very thin in order to reduce the effects of multiple scattering of the electron beam in the diamond radiator. Further studies are needed to determine what is causing the observed curvature and how it may be reduced, perhaps through additional post-processing steps or improved mounting techniques.

The above results clearly demonstrate that rocking curve imaging is a very powerful method for assessing the suitability of diamond crystals for use as CB radiators. The resulted $2 \mathrm{D}$ maps of rocking curve width as well as the rocking curve peak position can serve as a monitor of the crystal quality for the whole crystal and for local 
regions. It was confirmed by the measured variation of rocking curve widths across the samples studied that the defect distribution is non-uniform in these samples. For the thinnest diamond sample studied, crystal warping contributes significantly to the rocking curve width for the region to be sampled by the electron beam in the coherent bremsstrahlung process. Therefore it is essential that X-ray measurements to verify the suitability of a diamond for use as a CB radiator be performed after the diamond has been polished to its final thickness and mounted in its final fixture.

\section{Acknowledgements:}

This work is based upon research conducted at the Cornell High Energy Synchrotron Source (CHESS) which is supported by the National Science Foundation and the National Institutes of Health/National Institute of General Medical Sciences under NSF award DMR-0225180. One of the authors (G. Yang) would like to thank Thomas Jefferson lab for the financial support. 


\section{References}

[1] Ferrari, C., Koryta’' r, D. \& Kumar, J. (1997). Il Nuovo Cimento, 19D(2-4), 165-173.

[2] D.Sober et al., Nucl. Inst. and Meth., A440 263 (2000).

[3] J-O.Adler, V.Ganenko, L-J.Lindgren. Report 01/01 LUNFD6/(NFFR-3086)1-31/2001, Lund 2001

[4] D. Lohman et al., Nucl. Instr. Meth. A343, 494 (1994)

[5] U. Timm, Fortschritte der Physik 17 u765 (1969).

[6] J. D. Kellie et al, Nuclear instrument and Methods in Pphysics Research A 545 (2005) 164-180

[7] H. Hakopian et. al. GlueX technical note, GlueX Doc 1227.

[8] Albert et. al. Ap-plied Physics Letter 87, 194113 (2005)

[9] D. Hull, D. J. Bacon, Introduction to dislocation, London: Pergamon 1984 